\title{
Air Pollution and Mortality: Effect Modification by Personal Characteristics and Specific Cause of Death in a Case-Only Study
}

\author{
Hong Qiu ${ }^{\mathrm{a}}$, Linwei Tian ${ }^{\mathrm{b} *}$, Kin-fai Ho ${ }^{\mathrm{a}}$, Vivian C. Pun ${ }^{\mathrm{a}}$, Xiaorong Wang ${ }^{\mathrm{a}}$, Ignatius T.S. Yu
}

a:The Jockey Club School of Public Health and Primary Care, The Chinese University of

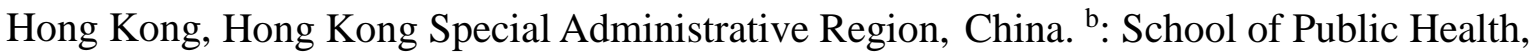
Li Ka Shing Faculty of Medicine, The University of Hong Kong.

Type of manuscript: Original Article

Running Title: Effect Modification on Pollution and Mortality Association

* Corresponding author:

Dr. Linwei Tian, School of Public Health, Li Ka Shing Faculty of Medicine, The University of Hong Kong, 21 Sassoon Road, Pokfulam, Hong Kong. Phone: (+852)2831 5071; Fax: (+852) 28559528 Email: linweit@hku.hk.

The authors declare we have no actual or potential competing financial interests.

Funding support: None

\section{Key Words:}

Air Pollution; Case-Only Study; Cause of Death; Effect Modifiers; Mortality

\section{Acknowledgements:}

The authors thank the Census and Statistical Department of Hong Kong for providing mortality data, the Hong Kong Environmental Protection Department for providing air pollution data, and the Hong Kong Observatory for providing temperature data. 


\begin{abstract}
Short-term effects of air pollution on mortality have been well documented in the literature worldwide. Less is known about which subpopulations are more vulnerable to air pollution. We conducted a case-only study in Hong Kong to examine the potential effect modification by personal characteristics and specific causes of death. Individual information of 402,184 deaths of non-external causes and daily mean concentrations of air pollution were collected from 2001 to 2011 . For a $10 \mu \mathrm{g} / \mathrm{m}^{3}$ increase of pollution concentration, people aged $>=65$ years (compared with younger ages) had a $0.9 \sim 1.8 \%$ additional increase in mortality related to $\mathrm{PM}, \mathrm{NO}_{2}$, and $\mathrm{SO}_{2}$. People dying from cardiorespiratory diseases (compared with other non-external causes) had a 1.6 2.3\% additional increase in $\mathrm{PM}$ and $\mathrm{NO}_{2}$ related mortality. Other subgroups that were particularly susceptible were females and those economically inactive. Lower socioeconomic status and causes of cardiorespiratory diseases would increase the likelihood of death associated with air pollution.
\end{abstract}

\title{
Key Words:
}

Air Pollution; Case-Only Study; Cause of Death; Effect Modifiers; Mortality

\section{Capsule Abstract:}

We conducted a case-only study to identify several personal characteristics and specific cardiorespiratory causes that vulnerable to air pollution related mortality. 


\section{Introduction}

Consistent strong evidence for a positive association between short-term air pollution exposure and daily mortality has been well documented worldwide (Katsouyanni et al., 1997; Samet et al., 2000; Wong et al., 2008b). Less is known about the modification for the pollution effect by factors measured at individual level. Identifying the modifiers regarding the susceptibility and vulnerability to pollution related mortality and morbidity would provide information for Environmental Protection Authorities to set health-related regulations with adequate margins of safety for sensitive individuals; and provide information for physicians to know about which population subgroups are most affected. Furthermore, understanding vulnerable populations may provide scientific evidence related to credible pathological mechanisms (Bell et al., 2013). Identification of sets of individuals who have an enhanced response to air pollution may suggest possible mechanisms of physiological assault, as well as provide data that can be used for more detailed risk assessment.

Modifiers at individual level on pollution associated mortality have been examined in previous studies (Bell et al., 2013; Cakmak et al., 2011; Forastiere et al., 2005; Ou et al., 2008; Peel et al., 2007; Qian et al., 2013; Ren et al., 2010; Zanobetti et al., 2000; Zanobetti and Schwartz, 2002; Zeka et al., 2006). There was strong and consistent evidence that elderly experience higher risk of pollution associated mortality (Bell et al., 2013; Cakmak et al., 2011; Forastiere et al., 2005; Zanobetti and Schwartz, 2002). There was some weak evidence that women have higher risks of dying from air pollution than men (Bell et al., 2013; Zanobetti et al., 2000; Zeka et al., 2006). Some studies examined the modification from socioeconomic status (Bell et al., 2013; Cakmak et al., 2011; Ou et al., 2008; Qian et al., 2013; Ren et al., 2010) and preexisting comorbidities or the secondary diagnoses (Forastiere et al., 2005; Peel et al., 2007; Qian et al., 2013; Zeka et al., 2006), however, the results were mixed. Most of these studies used case-crossover approach with integrating the individual information or time series Poisson model with stratified subgroup analyses to access the potential modification. As an alternative, Armstrong BG in 2003 firstly proposed and applied the case-only approach to access how the fixed factors modified the effects of time varying factors in time series study (Armstrong, 2003). After that, several studies used case-only analysis to identify the sensitive subpopulation who is more vulnerable to die from air pollution (Medina-Ramón and Schwartz, 2008; Wong et al., 2008a) or extreme temperatures (Medina-Ramón et al., 2006; Schwartz, 2005; Zanobetti et al., 2013). 
Being a simplified approach that reduces the vulnerability to model miss-specification bias (Armstrong, 2003; Zanobetti et al., 2013), case-only analysis has its advantages over the conventional time series analysis on accessing the modification. In the current study, we aimed to use a case-only analysis to identify the time invariant individual factors that confer susceptibility to air pollution associated mortality, including personal social-demographic characteristics and specific causes of death.

\section{Materials and Methods}

\section{Mortality Data}

Daily mortality data from January 2001 to December 2011 were obtained from Census and Statistical Department of Hong Kong. Individual information included personal characteristics such as age, sex, occupational status and marital status. Primary causes of death were coded in International Classification of Diseases, $10^{\text {th }}$ version. The primary causes of death examined in this study as potential modifiers included all diseases of circulatory system (ICD-10: I00-I99) and diseases of respiratory system (ICD-10: J00-J99). Some specific causes of death were also examined, including stroke (ICD-10: I60-I69), myocardial infarction (MI, ICD-10: I21, I22), congestive heart failure (CHF, ICD-10: I50), cardiac arrest (ICD-10: I46), chronic obstructive pulmonary disease (COPD, ICD-10: J40-J44, J47) and pneumonia (ICD-10: J12-J18).

\section{Air Pollution and Weather Data}

Air pollution concentrations from January 2001 to December 2011 were obtained from the Environmental Protection Department of Hong Kong. Hourly concentrations of four criteria air pollutants $\left(\mathrm{PM}_{10}, \mathrm{NO}_{2}, \mathrm{O}_{3}\right.$, and $\left.\mathrm{SO}_{2}\right)$ were monitored in 14 monitoring stations located in different districts of Hong Kong. Three roadside stations and one station on a remote island were excluded, leaving 10 general stations to generate daily mean concentrations. Hourly concentrations of $\mathrm{PM}_{2.5}$ were only monitored in three general stations during the study period that could be used in this study. We calculated daily 24-hr mean concentrations of $\mathrm{PM}_{10}$, $\mathrm{PM}_{2.5}, \mathrm{NO}_{2}, \mathrm{SO}_{2}$ and 8-hr (10:00-18:00) mean concentration of $\mathrm{O}_{3}$ for each monitoring station if at least 75 percent of hourly concentrations of each pollutant were available, and then averaged them across the available stations to denote the pollution concentrations in Hong Kong (Qiu et al., 2013). 
The daily minimum, mean and maximum temperature for the same period was obtained from the Hong Kong Observatory. Previous researchers defined the extremely hot days as those with a daily minimum temperature at or greater than the $99^{\text {th }}$ percentile of its distribution in a city. The extremely cold days were defined as those with a daily maximum temperature at or less than the $1^{\text {st }}$ percentile (Medina-Ramón et al., 2006; Schwartz, 2005). Considering the modification of the pollution-mortality association might be confounded by ambient temperature at more moderate levels (Guo et al., 2014), we defined the hot/cold days using the $95^{\text {th }}$ and $5^{\text {th }}$ percentiles as the cutoff points respectively.

\section{Analytic Method}

This is a case-only study to access how the association between air pollution and mortality was modified by the personal characteristics and the specific primary causes of death.

Originally proposed for use in studies of gene-environment interactions, case-only approach involves measuring the environmental factor and the genotype (modifier) of a series of cases, without controls (Hamajima et al., 1999; Khoury and Flanders, 1996). If the environmental factor is more often present in cases with a specific genotype than in other cases, then this is evidence that the gene predisposes to the environmental factor causing the disease. Crucially, this conclusion relies on the assumption that the genotype and environmental factor are not associated in the base population from which the cases arose. If there is departure from such independence assumption, the inference about the interaction with case-only design can be highly distorted (Albert et al., 2001). For time-series air pollution studies, there are only "cases", and no denominators or controls. The well accepted standardized statistical modeling methods that used in time series studies to examine the short-term effect of air pollution are generalized additive Poisson models (Peng et al., 2006) and the equivalent case-crossover analysis (Lu et al., 2008).

Considering a traditional Poisson model to examine a potential modifier on the acute effect of air pollution on death, researchers usually do the stratified analyses in different categories of interested modifier (Kan et al., 2008), or include an interaction term of such modifier and pollutant to model the number of deaths per day (Lipsett et al., 1997) as:

$$
\begin{aligned}
\log (E(Y)) & =\beta_{0}+\beta_{1} \text { pollutant }+\beta_{2} \text { modifier } \\
& +\beta_{3} \text { pollutant } \times \text { modifier }+ \text { confounders }
\end{aligned}
$$


If we are only interested in the potential modifying effect of a characteristic but not the main effect of air pollutant, $\beta_{3}$ in model (1) is the answer. Armstrong BG (Armstrong, 2003) and Schwartz J (Schwartz, 2005) proposed the use of the case-only approach to study modification by individual factors that are effectively time invariant (such as sex, social economic status (SES), or housing type) in time-series studies of effects of air pollution, weather or other time-varying factors. The underlying idea behind this approach is that if a time invariant factor increases the risk of dying on pollution exposure, a greater proportion of people who died of high levels of pollution would be expected to have this factor, compared with people who died of milder pollution exposure. Hence, if a characteristic is a risk modifier for death from pollution, then pollution exposure should be a predictor of the occurrence of that characteristic on death certificates using logistic regression (Schwartz, 2005) as:

$$
\text { logit }(\text { modifier }=1)=\alpha+\beta \times \text { air pollutant } \ldots
$$

The logistic regression model (2) using only the dead subjects predicts the presence of a modifier as the function of pollution which infers the interaction in the time series Poisson model (1) applying to the entire population. The validity of this approach depends on the assumption that the modifier and exposure are not associated in the base population that gave rise to the deaths. Because the personal characteristics vary among individuals but not fluctuate from day to day variation of air pollution, this assumption is clearly met in studies of air pollution. Armstrong also suggested that the assumption that modifiers are fixed in time could be relaxed to allow modifiers that change in time much more slowly than does the air pollution, such as age, chronic disease, and some long-term treatments (Armstrong, 2003). The case-only approach was proposed for analysis rather than for design, and the motivation is simplification of modeling and reduced vulnerability to model miss-specification bias.

There are still some modeling difficulties that the case-only approach does not simplify. It is critical to the argument for the validity of this approach that there are no other interactions of time-varying factors with time-fixed factors. These would confound the interaction of interest. The following two types of such interactions would almost inevitably confound:

1). Interaction of the putative modifier under investigation with other time-varying factors. For example, SES modifies, say, the effect of extreme temperature.

2). Interaction of the time-varying factor of interest with another time-fixed variable. For example, the pollution effect is modified by, say, age. 
Confounding interactions of type 1) could also be modeled in the case-only approach. For example, temperature levels could be entered as an additional indicator in the case-only model. The incorporation of interactions of type 2) seems less obvious (Armstrong, 2003).

Furthermore, as J Schwartz mentioned, because of the baseline seasonal pattern of mortality, the additional non-pollution related modifiers of risk by predisposing condition might be captured by a sine and cosine term with 365.24-day period (Schwartz, 2005). We therefore included an annual sine-cosine pair to sufficiently control for a seasonal component that might plausibly confound the modifier of interest and pollution.

So we included the interaction of the putative modifier under investigation with temperature and seasonal pattern that might confound the interaction of interest as a sensitivity analysis, in which the 'temperature' was a categorical indicator with three levels (0 for normal temperature, 1 for hot days and 2 for cold days) and the seasonal pattern was captured by an annual sine-cosine pair.

Binary logistic regression was repeatedly used to examine the modifier with two levels such as personal characteristics (age, gender, employment and marital status); multinomial logistic regression was used to examine the modifier with several categories such as specific diagnosed cause of death. All analyses were conducted in SPSS version 18.0.

\section{Results}

We included a total of 402,184 non-external causes of death in Hong Kong in this case-only analysis. Most of the deaths were age of 65 years and older (80.4\%) and economically inactive (90.6\%). A little more deaths were males (55.6\%) and never married/widowed/divorced (52.1\%). Diseases of circulatory and respiratory system accounted for $27.5 \%$ and $19.5 \%$ of the total non-external causes of death, respectively (Table-1). The temporal distribution of daily air pollution concentrations from 2001 to 2011 was displayed in time-series plots (Figure-1). The correlation between $\mathrm{PM}$ and $\mathrm{NO}_{2}$ was high with correlation coefficients 0.722 for $\mathrm{PM}_{10}$ and $\mathrm{NO}_{2}$, and 0.775 for $\mathrm{PM}_{2.5}$ and $\mathrm{NO}_{2}$, while the correlations between $\mathrm{PM}, \mathrm{NO}_{2}$ and $\mathrm{O}_{3}, \mathrm{SO}_{2}$ were low to moderate with correlation coefficients 0.211 to 0.556 .

Table-2 shows the individual characteristics that modify the effects of air pollution. For a 10 $\mu \mathrm{g} / \mathrm{m}^{3}$ increase of pollution concentration (average of lags 0 to 2 ), people aged $>=65$ years 
(compared with younger ages) had a $1.1 \%$ (95\% confidence interval: $0.8 \%$ to $1.4 \%$ ), $1.5 \%$ (1.1\% to $1.9 \%), 1.8 \%(1.3 \%$ to $2.2 \%)$ and $0.9 \%(0.2 \%$ to $1.7 \%)$ additional increase in mortality related to $\mathrm{PM}_{10}, \mathrm{PM}_{2.5}, \mathrm{NO}_{2}$, and $\mathrm{SO}_{2}$, respectively. Those economically inactive had a $1.7 \%(1.2 \%$ to $2.1 \%), 2.0 \%$ (1.4\% to $2.5 \%), 2.3 \%$ (1.7\% to $2.8 \%)$ and $6.3 \%$ (5.2\% to $7.5 \%$ ) additional increase in mortality related to $\mathrm{PM}_{10}, \mathrm{PM}_{2.5}, \mathrm{NO}_{2}$, and $\mathrm{SO}_{2}$, respectively. Other subgroups that were particularly susceptible were females with a marginally additional $0.2 \%$ to $0.3 \%$ increase in mortality related to particulate matter, and those never married/widowed/divorced with $1.6 \%$ decrease in $\mathrm{SO}_{2}$ related mortality than married. People with cardiorespiratory diseases (compared with other non-external causes) had a $1.6 \%(1.3 \%$ to $1.8 \%), 2.1 \%(1.7 \%$ to $2.4 \%)$, and $2.3 \%$ (2.0\% to $2.6 \%)$ additional increase in mortality related to $\mathrm{PM}_{10}, \mathrm{PM}_{2.5}$, and $\mathrm{NO}_{2}$, respectively. Sensitivity analyses with additionally control for the interactions with seasonal pattern (a sine and cosine term with 365.24-day period) and temperature levels did not change the results, showing the modification of individual characteristics on pollution effects were stable (Table-3). Modification of cardio-respiratory causes on $\mathrm{O}_{3}$ and $\mathrm{SO}_{2}$ was not found.

Table-4 shows the specific causes of death that modify the effects of $\mathrm{PM}$ and $\mathrm{NO}_{2}$. For a 10 $\mu \mathrm{g} / \mathrm{m}^{3}$ increase of pollution concentration (average of lags 0 to 2), cause of circulatory diseases (compared with non-cardiorespiratory causes) had a $1.9 \%$ (1.6\% to $2.2 \%$ ), $2.5 \%$ ( $2.1 \%$ to $2.9 \%$ ) and $2.8 \%$ (2.4\% to $3.2 \%$ ) additional increase in mortality related to $\mathrm{PM}_{10}$, $\mathrm{PM}_{2.5}$, and $\mathrm{NO}_{2}$, respectively. Cause of respiratory diseases (compared with non-cardiorespiratory causes) had a $0.7 \%$ ( $0.4 \%$ to $1.1 \%), 0.8 \%(0.4 \%$ to $1.2 \%)$ and $1.3 \%$ $(0.9 \%$ to $1.8 \%)$ increased risk of dying related to $\mathrm{PM}_{10}, \mathrm{PM}_{2.5}$, and $\mathrm{NO}_{2}$, respectively. Individuals diagnosed with cardiac arrest showed the highest increase in the risk of dying related to PM (additional 5.2 6.5\% increase). Subjects dying from other specific causes of death such as stroke, MI, CHF, COPD and pneumonia also showed the susceptibility to the $\mathrm{PM}$ and $\mathrm{NO}_{2}$ pollution exposure (Table-4).

\section{Discussion}

In this case-only study, we identified several subpopulations particularly vulnerable to air pollution. Elder and economically inactive subjects were especially susceptive to particulate matter $\left(\mathrm{PM}_{10}\right.$ or $\left.\mathrm{PM}_{2.5}\right), \mathrm{NO}_{2}$ and $\mathrm{SO}_{2}$ exposure; never married, widowed and divorced subjects were less pronounced to $\mathrm{PM}$ and $\mathrm{SO}_{2}$ exposure than married; females were a little 
more sensitive to PM exposure than males. Regarding specific causes of death, cardiorespiratory deaths showed a higher susceptibility to air pollution exposure, which was particularly noticeable for cardiac arrest deaths. Besides this, the increase in pollution associated deaths was higher for subjects dying from congestive heart failure, myocardial infarction, stroke, COPD and pneumonia.

There is consistent evidence in the literature that the risk of death associated with short-term air pollution exposure is higher in elder populations than in younger populations (Bell et al., 2013; Cakmak et al., 2011; Forastiere et al., 2005; Zanobetti and Schwartz, 2002). In addition to physiological changes that accompany age, older persons likely have different indoor/outdoor activity patterns, occupational exposures, and social networks. We found that particulate matter exposure risks were marginally higher for women than for men, which was consistent with a recent systematic review and meta-analysis showing the weak evidence that women have higher risks of death from PM (Bell et al., 2013) and some previous studies (Zanobetti et al., 2000; Zeka et al., 2006). Gender different risks may result from differences in physiology, exposure patterns, and /or activity patterns.

Economically inactive subjects were vulnerable to the risk of pollution associated mortality except ozone. In previous studies based on individual-level data, risk estimates higher for those with lower employment status, for unemployed persons compared with white-collar employees (Cakmak et al., 2011), and for blue-collar workers or never employed persons compared with white-collar workers (Ou et al., 2008) have been reported. Employment status relates to social economic status, which may modify the air pollution associated death risks through differences in access to health care, baseline health status, occupational exposure, and nutrition (Bell et al., 2013; Forastiere et al., 2007). However, besides the unemployed, the "economically inactive" category also refers to the retired persons, whose modified effect may be contaminated by the older age. This is the first study showing those never married/widowed/divorced were less susceptible to $\mathrm{PM}$ and $\mathrm{SO}_{2}$ related mortality than married. In other words, married couples were vulnerable to $\mathrm{PM}$ and $\mathrm{SO}_{2}$ exposure, which may possibly due to the different outdoor activity patterns. There are only a few studies up to date to examine the modification of marital status on pollution associated mortality, and no consistent evidence of modification by marital status was found (Krewski et al., 2005; Ren et al., 2010). 
We found the susceptibility of cardiorespiratory causes of death to air pollution. Individuals diagnosed with cardiorespiratory diseases showed higher increased risk of dying related to $\mathrm{PM}$ and $\mathrm{NO}_{2}$. Subjects dying from some specific causes including cardiac arrest, stroke, myocardial infarction, congestive heart failure, COPD and pneumonia showed greater susceptibility to air pollution exposure. Similar modification of the medical conditions at individual level has been examined in several previous studies. Work by Zanobetti et al. focused on subgroups of patients with cardio-respiratory disorders and showed that those with acute respiratory infections and with defects in the electrical control of heart rate and rhythm appeared to be at particular risk of adverse effects on exposure to particles (Zanobetti et al., 2000). Zeka et al. reported that secondary pneumonia enhanced $\mathrm{PM}_{10}$-stroke mortality (Zeka et al., 2006). Forastiere et al. suggested the effect modification for people with COPD on air pollution associated out-of-hospital coronary death (Forastiere et al., 2005). Peel et al. provided further evidence of increased susceptibility to adverse cardiovascular events associated with air pollution among persons with COPD (Peel et al., 2007). Qian et al. reported that comorbid cardiac diseases increased the risk of ischemic-stroke mortality in relation to air pollution exposure, especially $\mathrm{NO}_{2}$ (Qian et al., 2013). Mechanisms that may explain such a marked increase in cardiorespiratory deaths with exposure to high pollution concentrations have been postulated. Those suffering from cardio-respiratory disorders may have an already inflamed airway and the impaired clearance of particulates which lead to the increased deposition of fine PM (Brown et al., 2002).

The conventional approach to investigate the modification of personal characteristics and pre-existing comorbidities is by inclusion of interaction terms in the regression model of outcome on the exposure and modifier, or by carrying out separate regressions on strata with different modifier status (Kan et al., 2008; Lipsett et al., 1997; Peel et al., 2007; Qian et al., 2013; Zanobetti et al., 2000; Zeka et al., 2006). However, the case-only approach provides important advantages over traditional analyses, including reduction of potential confounding by variables typically associated with mortality, simplification of modeling, and reduction of results sensitivity to model misspecification bias (Armstrong, 2003; Zanobetti et al., 2013). Of particular interest, the long term trend and periodicity in mortality whose modeling is quite complex, drops out in case-only approach. It was encouraging that in this study, seasonal pattern and temperature levels did not appear to confound the interaction of interested modifiers and pollution. Additionally control for the interactions with season (a sine and cosine term with 365.24-day period) and temperature levels did not change the 
results and show the modification of individual characteristics on pollution effects were stable. Although such findings need to be confirmed in more locations, including areas with different climates, they support the use of the case-only approach to examine susceptibility. One limitation of this study is that we could not link the death data to the previous medical records of the decedents, so that we were unable to abstract the pre-existing comorbidities for each subject. Although the subjects died of cardiorespiratory diseases may probably have the pre-existing chronic cardiorespiratory diseases, modification of the specific primary causes of death identified in this study may still not exactly represent the modification of the pre-existing comorbidities. Secondary, air pollution concentrations averaged from general monitoring stations were assigned to all the decedents. This is the inherent ecological error and may introduce some misclassification of the pollution exposure, fortunately, such bias is non-differential. Another limitation came from the case-only study design, which could not estimate the main effect of the pollution but only the effect modification by certain characteristics. However, the main effects of air pollution on mortality have been well studied in Hong Kong (Wong et al., 2008a, 2008b, 2002) and documented in the literature, identifying the modification is the main purpose of this study. Finally, the modification of 'Economically inactive' on the pollution associated mortality presents the different direction for ozone from other pollutants. These may probably due to the complex associations among pollutants in the air pollution mixture, and the possible different indoor/outdoor and daytime/night time activity patterns in the different demographic groups, which needs further studied.

In conclusion, we identified several personal characteristics (including old age, female and economically inactive) and specific cardiorespiratory mortality causes that particularly susceptible to air pollution exposure. Information on vulnerable subpopulations would help guide more detailed risk assessment and provide a better understanding of air pollution toxicity. 


\section{References:}

Albert, P., Ratnasinghe, D., Tangrea, J., Wacholder, S., 2001. Limitations of the case-only design for identifying gene-environment interactions. Am. J. Epidemiol. 154, 687-693.

Armstrong, B.G., 2003. Fixed factors that modify the effects of time-varying factors: applying the case-only approach. Epidemiology 14, 467-72.

Bell, M.L., Zanobetti, A., Dominici, F., 2013. Evidence on vulnerability and susceptibility to health risks associated with short-term exposure to particulate matter: a systematic review and meta-analysis. Am. J. Epidemiol. 178, 865-76.

Brown, J.S., Zeman, K.L., Bennett, W.D., 2002. Ultrafine particle deposition and clearance in the healthy and obstructed lung. Am. J. Respir. Crit. Care Med. 166, 1240-7.

Cakmak, S., Dales, R.E., Rubio, M.A., Vidal, C.B., 2011. The risk of dying on days of higher air pollution among the socially disadvantaged elderly. Environ. Res. 111, 388-93.

Forastiere, F., Stafoggia, Ã.M., Tasco, C., Picciotto, S., Agabiti, N., Cesaroni, G., Perucci, C.A., 2007. Socioeconomic Status, Particulate Air Pollution, and Daily Mortality: Differential Exposure or Differential Susceptibility. Am. J. Ind. Med. 216, 208-216.

Forastiere, F., Stafoggia, M., Picciotto, S., Bellander, T., D’Ippoliti, D., Lanki, T., von Klot, S., Nyberg, F., Paatero, P., Peters, A., Pekkanen, J., Sunyer, J., Perucci, C. a, 2005. A case-crossover analysis of out-of-hospital coronary deaths and air pollution in Rome, Italy. Am. J. Respir. Crit. Care Med. 172, 1549-55.

Guo, Y., Gasparrini, A., Armstrong, B., Li, S., Tawatsupa, B., Tobias, A., Lavigne, E., de Sousa Zanotti Stagliorio Coelho, M., Leone, M., Pan, X., Tong, S., Tian, L., Kim, H., Hashizume, M., Honda, Y., Guo, Y.-L.L., Wu, C.-F., Punnasiri, K., Yi, S.-M., Michelozzi, P., Saldiva, P.H.N., Williams, G., 2014. Global variation in the effects of ambient temperature on mortality: a systematic evaluation. Epidemiology 25, 781-9.

Hamajima, N., Yuasa, H., Matsuo, K., Kurobe, Y., 1999. Detection of gene-environment interaction by case-only studies. Jpn. J. Clin. Oncol. 29, 490-3.

Kan, H., London, S.J., Chen, G., Zhang, Y., Song, G., Zhao, N., Jiang, L., Chen, B., 2008. Season, sex, age, and education as modifiers of the effects of outdoor air pollution on daily mortality in Shanghai, China: The Public Health and Air Pollution in Asia (PAPA) Study. Environ. Health Perspect. 116, 1183-8.

Katsouyanni, K., Touloumi, G., Spix, C., Schwartz, J., Balducci, F., Medina, S., Rossi, G., Wojtyniak, B., Sunyer, J., Bacharova, L., Schouten, J.P., Ponka, A., Anderson, H.R., 1997. Short term effects of ambient sulphur dioxide and particulate matter on mortality 
in 12 European cities: results from time series data from the APHEA project. BMJ 314, $1658-63$.

Khoury, M.J., Flanders, W.D., 1996. Nontraditional epidemiologic approaches in the analysis of gene-environment interaction: case-control studies with no controls! Am. J. Epidemiol. 144, 207-213.

Krewski, D., Burnett, R.T., Goldberg, M., Hoover, K., Siemiatycki, J., Abrahamowicz, M., Villeneuve, P.J., White, W., 2005. Reanalysis of the Harvard Six Cities Study, part II: sensitivity analysis. Inhal. Toxicol. 17, 343-53.

Lipsett, M., Hurley, S., Ostro, B., 1997. Air pollution and emergency room visits for asthma in Santa Clara County, California. Environ. Health Perspect. 105, 216-22.

Lu, Y., Symons, J.M., Geyh, A.S., Zeger, S.L., 2008. An approach to checking case-crossover analyses based on equivalence with time-series methods. Epidemiology $19,169-75$.

Medina-Ramón, M., Schwartz, J., 2008. Who is more vulnerable to die from ozone air pollution? Epidemiology 19, 672-9.

Medina-Ramón, M., Zanobetti, A., Cavanagh, D.P., Schwartz, J., 2006. Extreme Temperatures and Mortality: Assessing Effect Modification by Personal Characteristics and Specific Cause of Death in a Multi-City Case-Only Analysis. Environ. Health Perspect. 114, 1331-1336.

Ou, C.-Q., Hedley, A.J., Chung, R.Y., Thach, T.-Q., Chau, Y.-K., Chan, K.-P., Yang, L., Ho, S.-Y., Wong, C.-M., Lam, T.-H., 2008. Socioeconomic disparities in air pollution-associated mortality. Environ. Res. 107, 237-44.

Peel, J.L., Metzger, K.B., Klein, M., Flanders, W.D., Mulholland, J. a, Tolbert, P.E., 2007. Ambient air pollution and cardiovascular emergency department visits in potentially sensitive groups. Am. J. Epidemiol. 165, 625-33.

Peng, R.D., Dominici, F., Louis, T. a., 2006. Model choice in time series studies of air pollution and mortality. J. R. Stat. Soc. Ser. A (Statistics Soc. 169, 179-203.

Qian, Y., Zhu, M., Cai, B., Yang, Q., Kan, H., Song, G., Jin, W., Han, M., Wang, C., 2013. Epidemiological evidence on association between ambient air pollution and stroke mortality. J. Epidemiol. Community Health 67, 635-40.

Qiu, H., Yu, I.T.-S., Wang, X., Tian, L., Tse, L.A., Wong, T.W., 2013. Cool and dry weather enhances the effects of air pollution on emergency IHD hospital admissions. Int. J. Cardiol. 168, 500-5. 
Ren, C., Melly, S., Schwartz, J., 2010. Modifiers of short-term effects of ozone on mortality in eastern Massachusetts--a case-crossover analysis at individual level. Environ. Health 9,3 .

Samet, J., Dominici, F., Curriero, F., Coursac, I., Zeger, S., 2000. Fine particulate air pollution and mortality in 20 U.S. cities, 1987-1994. N. Engl. J. Med. 343, 1742-9.

Schwartz, J., 2005. Who is Sensitive to Extremes of Temperature? Epidemiology 16, 67-72.

Wong, C.-M., Ou, C.-Q., Chan, K.-P., Chau, Y.-K., Thach, T.-Q., Yang, L., Chung, R.Y.-N., Thomas, G.N., Peiris, J.S.M., Wong, T.-W., Hedley, A.J., Lam, T.-H., 2008a. The effects of air pollution on mortality in socially deprived urban areas in Hong Kong, China. Environ. Health Perspect. 116, 1189-94.

Wong, C.-M., Vichit-Vadakan, N., Kan, H., Qian, Z., 2008b. Public Health and Air Pollution in Asia (PAPA): a multicity study of short-term effects of air pollution on mortality. Environ. Health Perspect. 116, 1195-202.

Wong, T.W., Tam, W.S., Yu, T.S., Wong, a H.S., 2002. Associations between daily mortalities from respiratory and cardiovascular diseases and air pollution in Hong Kong, China. Occup. Environ. Med. 59, 30-35.

Zanobetti, a, Schwartz, J., Gold, D., 2000. Are there sensitive subgroups for the effects of airborne particles? Environ. Health Perspect. 108, 841-5.

Zanobetti, A., O’Neill, M.S., Gronlund, C.J., Schwartz, J.D., 2013. Susceptibility to mortality in weather extremes: effect modification by personal and small-area characteristics. Epidemiology 24, 809-19.

Zanobetti, A., Schwartz, J., 2002. Cardiovascular damage by airborne particles: are diabetics more susceptible? Epidemiology 13, 588-92.

Zeka, A., Zanobetti, A., Schwartz, J., 2006. Individual-level modifiers of the effects of particulate matter on daily mortality. Am. J. Epidemiol. 163, 849-59. 


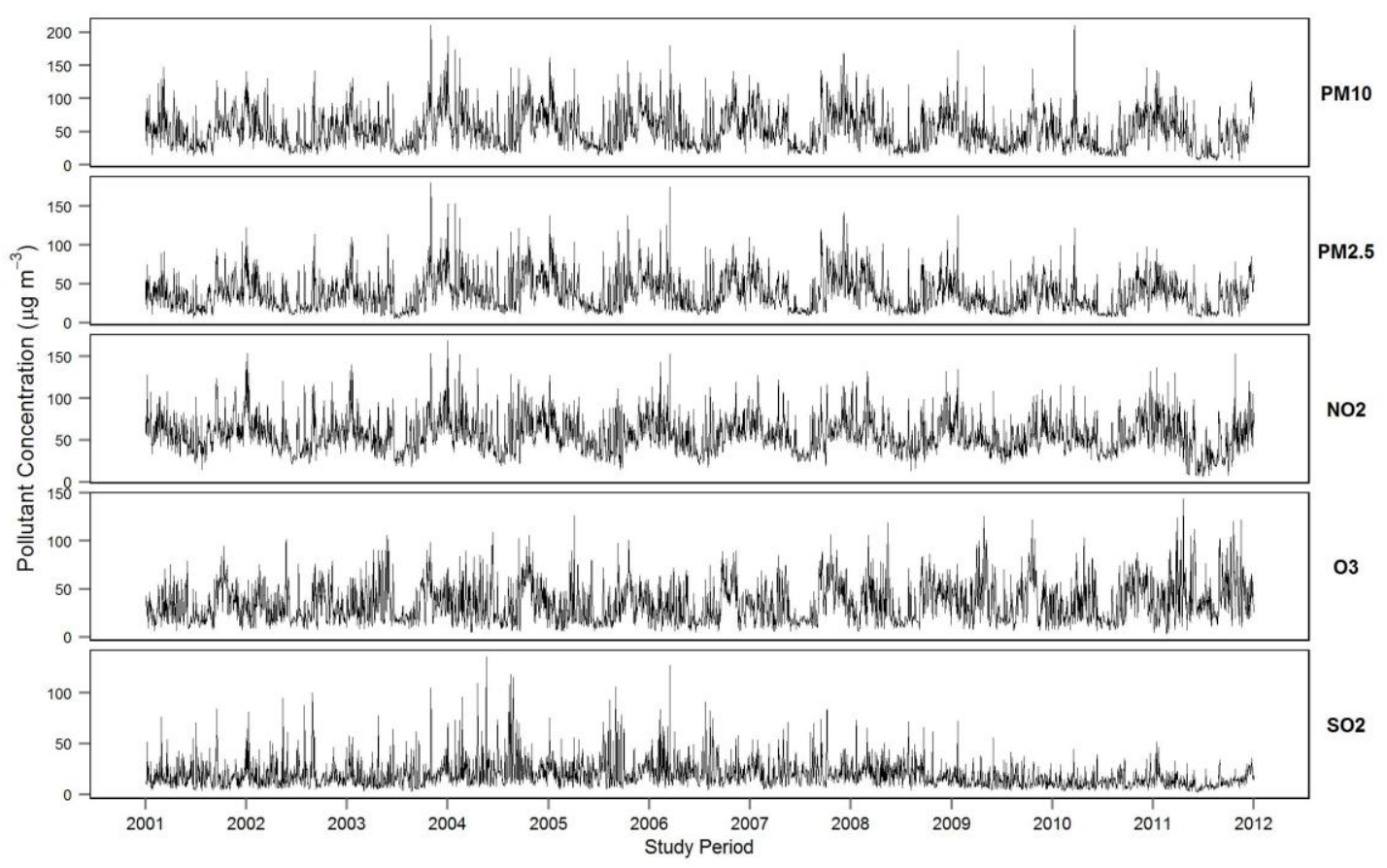

Figure-1 Temporal Distribution of Daily Air pollution Concentrations in Hong Kong, 2001-2011 
Table-1 Descriptive Statistics for Non-external Causes of Death (2001-2011)

\begin{tabular}{lcc}
\hline \multicolumn{1}{c}{ Category } & Number & Percentage (\%) \\
\hline Total & 402,184 & 100.0 \\
Age & & \\
$\quad<65$ yrs & 78,673 & 19.6 \\
$\quad>=65$ yrs & 323,511 & 80.4 \\
Sex & & \\
$\quad$ Male & 223,628 & 55.6 \\
Female & 178,556 & 44.1 \\
Occupational Status & & \\
$\quad$ With occupation & 37,910 & 9.4 \\
$\quad$ Economically inactive & 364,274 & 90.6 \\
Married Status & & 47.9 \\
$\quad$ Married & 192,504 & 38.0 \\
Never married/Widowed/Divorced & 153,096 & 14.1 \\
Unknown & 56,584 & \\
Cause of death & & 27.5 \\
All cardiovascular diseases & 110,609 & 9.3 \\
$\quad$ Stroke & 37,321 & 5.1 \\
$\quad$ Myocardial Infarction & 20,525 & 2.0 \\
Congestive Heart Failure & 7,910 & 0.1 \\
$\quad$ Cardiac Arrest & 284 & 19.5 \\
All respiratory diseases & 78,508 & \\
$\quad$ COPD & 19,811 & \\
$\quad$ Pneumonia & 49,990 & \\
\hline
\end{tabular}


Table-2 Additional Percent Change in Pollution-Related Mortality for Individuals with Potential Effect Modifier*

\begin{tabular}{lccccc}
\hline Modifier & \multicolumn{1}{c}{$\mathbf{P M}_{\mathbf{1 0}}$} & \multicolumn{1}{c}{$\mathbf{P M}_{\mathbf{2 . 5}}$} & \multicolumn{1}{c}{$\mathbf{N O}_{\mathbf{2}}$} & \multicolumn{1}{c}{$\mathbf{O}_{\mathbf{3}}$} & \multicolumn{1}{c}{$\mathbf{S O}_{\mathbf{2}}$} \\
\hline Age of 65yrs and older & $1.1(0.8,1.4)$ & $1.5(1.1,1.9)$ & $1.8(1.3,2.2)$ & $-0.1(-0.5,0.4)$ & $0.9(0.2,1.7)$ \\
Female & $0.2(0.0,0.5)$ & $0.3(0.0,0.6)$ & $0.2(-0.1,0.5)$ & $0.3(-0.1,0.6)$ & $0.4(-0.2,1.0)$ \\
Economically Inactive & $1.7(1.2,2.1)$ & $2.0(1.4,2.5)$ & $2.3(1.7,2.8)$ & $-2.5(-3.1,-2.0)$ & $6.3(5.2,7.5)$ \\
Never married/Widowed & $-0.2(-0.5,0.0)$ & $-0.3(-0.7,0.0)$ & $-0.1(-0.4,0.3)$ & $0.1(-0.2,0.5)$ & $-1.6(-2.2,-1.0)$ \\
/Divorced & & & & & \\
Cardio-respiratory causes & $1.6(1.3,1.8)$ & $2.1(1.7,2.4)$ & $2.3(2.0,2.6)$ & $0.0(-0.3,0.4)$ & $0.2(-0.4,0.8)$ \\
\hline
\end{tabular}

*: Effect modification was estimated for a $10 \mu \mathrm{g} / \mathrm{m}^{3}$ increase of pollution concentration averaged of lags 0 to 2 .

Table-3 Sensitivity Analysis with Additionally Control for Season and Temperature *

\begin{tabular}{lccccc}
\hline Modifier & \multicolumn{1}{c}{$\mathbf{P M}_{\mathbf{1 0}}$} & \multicolumn{1}{c}{$\mathbf{P M}_{\mathbf{2 . 5}}$} & \multicolumn{1}{c}{$\mathbf{N O}_{\mathbf{2}}$} & \multicolumn{1}{c}{$\mathbf{O}_{\mathbf{3}}$} & \multicolumn{1}{c}{$\mathbf{S O}_{\mathbf{2}}$} \\
\hline Age of 65yrs and older & $1.0(0.7,1.3)$ & $1.3(0.9,1.7)$ & $1.7(1.3,2.1)$ & $0.1(-0.4,0.5)$ & $1.1(0.3,1.9)$ \\
Female & $0.2(0.0,0.5)$ & $0.3(0.0,0.6)$ & $0.2(-0.1,0.5)$ & $0.3(0.0,0.7)$ & $0.4(-0.2,1.0)$ \\
Economically Inactive & $1.7(1.3,2.1)$ & $2.0(1.4,2.6)$ & $2.3(1.8,2.9)$ & $-2.5(-3.1,-2.0)$ & $6.4(5.3,7.6)$ \\
Never married/Widowed & $-0.3(-0.5,0.0)$ & $-0.4(-0.7,0.0)$ & $-0.1(-0.5,0.3)$ & $0.2(-0.2,0.6)$ & $-1.6(-2.2,-1.0)$ \\
/Divorced & & & & & \\
Cardio-respiratory causes & $1.4(1.2,1.6)$ & $1.8(1.5,2.1)$ & $2.2(1.9,2.6)$ & $0.2(-0.2,0.5)$ & $0.4(-0.2,1.0)$ \\
\hline
\end{tabular}

*: Effect modification was estimated for a $10 \mu \mathrm{g} / \mathrm{m}^{3}$ increase of pollution concentration averaged of lags 0 to 2 .

The temperature were controlled by a categorical indicator with three levels ( 0 for normal temperature, 1 for hot days and

2 for cold days) and the seasonal pattern was captured by an annual sine-cosine pair with 365.24-day period.

Table-4 Additional Percent Change in Pollution-Related Mortality for Specific Cause of Death*

\begin{tabular}{lccc}
\hline Cause of Death & $\mathbf{P M}_{\mathbf{1 0}}$ & $\mathbf{P M}_{\mathbf{2 . 5}}$ & $\mathbf{N O}_{\mathbf{2}}$ \\
\hline All Circulatory Diseases & $1.9(1.6,2.2)$ & $2.5(2.1,2.9)$ & $2.8(2.4,3.2)$ \\
Stroke & $1.4(0.9,1.8)$ & $1.9(1.3,2.4)$ & $2.1(1.5,2.7)$ \\
Myocardial Infarction & $1.9(1.3,2.5)$ & $2.4(1.7,3.2)$ & $3.2(2.4,4.0)$ \\
Congestive Heart Failure & $2.1(1.3,3.0)$ & $2.9(1.8,4.1)$ & $2.8(1.5,4.0)$ \\
Cardiac Arrest & $5.2(0.9,9.7)$ & $6.5(0.6,12.8)$ & $6.0(-0.4,12.8)$ \\
All Respiratory Diseases & $0.7(0.4,1.1)$ & $0.8(0.4,1.2)$ & $1.3(0.9,1.8)$ \\
COPD & $1.1(0.5,1.7)$ & $1.2(0.4,1.9)$ & $2.2(1.4,3.0)$ \\
Pneumonia & $0.5(0.2,0.9)$ & $0.5(0.0,1.0)$ & $0.9(0.4,1.4)$ \\
\hline
\end{tabular}

*: Effect modification was estimated for a $10 \mu \mathrm{g} / \mathrm{m}^{3}$ increase of pollution concentration averaged of lags 0 to 2 .

The temperature were controlled by a categorical indicator with three levels ( 0 for normal temperature, 1 for hot days and 2 for cold days) and the seasonal pattern was captured by an annual sine-cosine pair with 365.24-day period. 